\title{
Die Grenzen der Selbstverpflichtung
}

Die meisten NGOs und Gewerkschaften waren stets skeptisch gegenüber freiwilligen Selbstverpflichtungen der Wirtschaft. Diese Skepsis hat ihren Ursprung nicht in der ideologischen Ablehnung privater Eigeninitiative oder einer blinden Staatsgläubigkeit. Sie gründet vielmehr in den Erfahrungen mit der alltäglichen Praxis vieler Unternehmen. Allzu oft besteht bis heute eine erhebliche Kluft zwischen den verbalen Verpflichtungen der Unternehmen zu ökologischem und sozialem Handeln und den realen Auswirkungen ihrer Aktivitäten auf Menschen und Umwelt.

$\mathrm{D}$

Von Jens Martens ie Hoffnung auf das freiwillige Engagement von Unternehmen basiert auf dem Missverständnis, dass die Verwirklichung sozialer, ökologischer und menschenrechtlicher Standards automatisch im Interesse der Unternehmen und ihrer Eigentümer liegt. Dies ist angesichts des globalisierten Wettbewerbsdrucks und der wachsenden Macht institutioneller Anleger mit ihren hohen Renditeerwartungen keineswegs der Fall. Es existiert in den Unternehmensstrategien ein Zielkonflikt zwischen den kurzfristigen wirtschaftlichen und den langfristigen gesellschaftlichen Interessen. Angesichts abstïrzender Aktienkurse und weltweiter Rezessionstendenzen behalten in diesem Konflikt die wirtschaftlichen Interessen der Shareholder, mikroökonomisch durchaus rational, meist die Oberhand (1).

\section{Verbindliche Kriterien unabdingbar}

Dennoch lehnen manche NGOs freiwillige Selbstverpflichtungen der Wirtschaft nicht grundsätzlich ab. In einer gemeinsamen Stellungnahme an die Kommission für nachhaltige Entwicklung (CSD) listete eine Gruppe von Umwelt- und Entwicklungs-NGOs sieben Kriterien auf, die erfüllt sein müssen, damit freiwillige Selbstverpflichtungen „einen positiven Beitrag zur nachhaltigen Entwicklung leisten" (2):

- Substanz: Klare Sprache und Zielsetzung, Fokus auf zentrale Themen, keine Doppeldeutigkeiten und Schlupflöcher.

- Partizipation: Beteiligung aller betroffenen „Stakeholder“.

- Motivation: Ausreichende Anreize zur Gewährleistung der freiwilligen Erfüllung.
- Integration: Verankerung sozialer und ökologischer Werte in Firmenzielen, Berücksichtigung in Instrumenten der Erfolgskontrolle.

- Transparenz: Rechtzeitige und inhaltlich angemessene Information über Produkte und Produktionsverfahren.

- Unabhängige Verifizierung: Bestätigung durch Dritte.

- Verantwortlichkeit/Rechenschaftspflicht: Verankerung von Sanktionsmechanismen.

Beurteilt man die innerbetrieblichen Verhaltenskodizes der Unternehmen anhand dieses Kriterienkataloges, ist festzustellen, dass kein einziger uns bekannter Kodex alle sieben Kriterien erfüllt. In der Regel ist weder die Partizipation der Betroffenen noch eine unabhängige Verifizierung vorgesehen. Konsequenterweise nutzen NGOs und Gewerkschaften diese Verhaltenskodizes als Referenzrahmen in der Regel nicht.

Immer mehr Unternehmen sehen den begrenzten Nutzen der ,ersten Generation“ von unilateralen Selbstverpflichtungen - nicht zuletzt für die eigene Öffentlichkeitsarbeit - und konzentrieren sich inzwischen auf weitergehende Initiativen mit stärkerer Legitimationskraft.

\section{Schwächen von Stakeholder- Ansätzen}

Dazu zählen in erster Linie die sogenannten Multistakeholder-Ansätze, die in den letzten Jahren stark zugenommen haben. Aber auch sie weisen eine Reihe struktureller Schwächen und Grenzen auf:

- Begrenzte Zahl: Trotz des Booms der letzten Jahre ist die Zahl der Initiativen und der beteiligten Unternehmen verglichen mit ihrer weltweiten Zahl gering. UNCTAD schätzt die Zahl transnationaler Unternehmen auf 60.000, die ihrer Tochter- firmen auf über 700.000. An MultistakeholderInitiativen sind insgesamt nur einige hundert Unternehmen beteiligt. Zumeist sind es Unternehmen, die auf Konsumentendruck reagieren (Kaufhauskette, Sportartikelindustrie), deren Produktion erhebliche Risiken birgt (Chemieindustrie), oder in denen Arbeitnehmerinteressen besonders gut gewerkschaftlich organisiert sind (Volkswagen).

- Begrenzte Beteiligung: Sowohl die Breite als auch die Quantität zivilgesellschaftlicher Mitwirkung in Multistakeholder-Initiativen sind begrenzt. Zivilgesellschaftliche Gruppen aus Entwicklungsländern sind wie etwa im SA 8000 meist unterrepräsentiert. In den Globalen Rahmenabkommen sind NGOs und lokale Gruppen generell nicht beteiligt. Lediglich einzelne NGOs und Gewerkschaften spielen eine herausragende Rolle, beispielsweise der WWF beim Marine Stewardship Council (MSC) oder die Internationalen Gewerkschaftsdachverbände bei den globalen Rahmenabkommen mit einzelnen Unternehmen.

- Begrenzter Fokus: Der thematische Fokus der einzelnen Initiativen ist sehr selektiv. Die einen verfolgen vor allem ökologische Aspekte, die anderen konzentrieren sich auf die Verwirklichung der Kernarbeitsnormen der ILO. Damit reflektieren die Initiativen in der Regel nur Teilaspekte unternehmerischer Verantwortung: Unternehmen picken sich die Verantwortungsbereiche heraus, in denen Engagement ihnen am wenigsten weh tut. Es handelt sich somit nicht um einen kohärenten Ansatz nachhaltiger Entwicklung, der ökologische, soziale, menschenrechtliche und ökonomische Ziele gleichermaßen umfasst.

- Begrenzte Überwachung: Die Einhaltung der freiwilligen Selbstverpflichtungen wird in sehr unterschiedlichem Umfang überwacht. Während manche Initiativen, wie etwa der Forest Stewardship Council, Zertifizierung und Auditing durch kommerzielle Firmen vorsehen, beschränken sich andere, wie einige globale Rahmenabkommen, auf die innerbetriebliche Kontrolle.

Manche, wie die Global Reporting Initiative, verzichten vollständig auf Prüfung. Der Einsatz kommerzieller Audit- und Zertifizierungsfirmen wird von NGOs skeptisch beurteilt. Sie selbst haben aber meist nicht die Kapazitäten zur systematischen Übernahme einer watchdog-Funktion. Effektiver sind möglicherweise Beschwerdeverfahren, die eine unabhängige Instanz und wirksame Sanktionsmechanismen erfordern. Über beides verfügen Multistakeholder-Initiativen in der Regel nicht. 
Im positiven Sinne können Multistakeholder-Initiativen Trendsetter-Funktion haben, indem sie in Bezug auf soziale, ökologische und menschenrechtliche Standards über die bestehenden gesetzlichen Regeln hinausgehen. Aber erst wenn alle Unternehmen dem gesetzten Trend folgen, kann eine positive Breitenwirkung erzielt werden. Dies geschieht erfahrungsgemäß nur, wenn staatliche oder zwischenstaatliche Institutionen die erforderlichen Regeln setzen. Somit sind hier die Grenzen der Freiwilligkeit erreicht.

Die OECD-Leitsätze für Multinationale Unternehmen zeigen, in welche Richtung internationale Regeln für Unternehmensverantwortung gehen können. Sie gelten für alle Unternehmen mit Sitz in den 33 Unterzeichnerstaaten. Sie umfassen ein breites Themenspektrum, das ökologische und arbeitsrechtliche Fragen einschließt. Und sie verfügen über einen, wenn auch rudimentären, Beschwerdemechanismus. Gravierende Schwächen sind die vage Formulierung der Unternehmenspflichten, die Abhängigkeit des Beschwerdeverfahrens von der Kooperationsbereitschaft der zuständigen nationalen Kontaktstellen und das Fehlen von Sanktions- und Entschädigungsverfahren bei Verletzung der Leitsätze.

\section{- Verankerung in nationalem Recht}

Die „Draft Norms on the Responsibilities of Transnational Corporations and other Business Enterprises with Regard to Human Rights" der UNUnterkommission zur Förderung und zum Schutz der Menschenrechte haben weitergehendes Potenzial(3). Insbesondere wenn sie mit nationalen Rechtsinstrumenten verknüpft und durch internationale Instrumente ergänzt würden. Derzeit kommt der Entwurf dieser Normen den Forderungen von NGOs und Gewerkschaften am nächsten. Zugleich steht er im Einklang mit dem Beschluss des Johannesburg-Gipfels, die Verantwortlichkeiten und Pflichten von Unternehmen unter anderem durch die Entwicklung zwischenstaatlicher Abkommen und internationaler Initiativen aktiv zu fördern.

Aus ihren bisherigen Erfahrungen mit freiwilligen Unternehmensaktivitäten und dem Engagement der Regierungen in diesem Bereich haben NGOs Konsequenzen gezogen. Bei internationalen Treffen und Strategiedebatten, wie zuletzt während des Weltsozialforums in Porto Alegre, zeichnete sich eine „Mehr-Ebenen-Strategie“ ab:

- Verstärkte Unterstützung lokaler Gruppen in ihrer Auseinandersetzung mit Transnationalen Unternehmen (TNCs). Dies schließt traditionelle
Formen der Öffentlichkeitsarbeit und des „naming and shaming" bis hin zu Boykotts ebenso ein, wie die aktivere Nutzung von Beschwerdemöglichkeiten und nationalen Klageverfahren. Eine Grundvoraussetzung für eine ,extraterritoriale" Anwendung nationaler Rechtsinstrumente gegenüber TNCs ist der bessere weltweite Informationsfluss zwischen den Gruppen in Nord und Süd.

- Verstärktes Eintreten für verbindliche Unternehmensregeln auf der nationalen und regionalen Ebene. Beispiele sind der britische Vorschlag für ein Gesetz zur Unternehmensverantwortung (,Corporate Responsibility Bill“) aus dem Jahr 2002 und der Vorschlag des Europäischen Parlaments für einen Verhaltenskodex für in Entwicklungsländern tätige europäische Unternehmen (4).

- Weiterentwicklung internationaler ,TrendsetterInitiativen " zwischen fortschrittlichen Unternehmen, NGOs und Gewerkschaften. Dies kann insbesondere dort sinnvoll sein, wo kurzfristige zwischenstaatliche Lösungen nicht in Sicht sind.

- Fortsetzung der internationalen Kampagne für verbindliche Unternehmensregeln. Zentrales Ziel vieler NGOs und Gewerkschaften ist weiterhin die Verankerung verbindlicher Unternehmensregeln auf der globalen Ebene, unter anderem mit den Draft Norms der UN-Unterkommission als Ausgangspunkt. Neben dem langfristigen Ziel einer Rahmenkonvention zur Unternehmensverantwortung geht es kurzfristig vor allem um Fortschritte in Teilbereichen. Beispiel wären ein internationales Umwelthaftungsrecht oder die verbindliche Offenlegung aller Zahlungsflüsse von Unternehmen an Regierungen.

\section{Thema Governance in Ökologisches Wirtschaften}

Vergangene Ausgaben

\section{2/1998 Global Governance} und Umweltpolitik

\section{3-4/2001 Governancestrukturen fuir Nachbaltigkeit}

Zu beziehen bei: CONSODATA ONE-TO-ONE Semmelweisstr. 8, 82152 Planegg Fon 089/85709-155, Fax -131

E-Mail: kontakł@oekom.de
In den internationalen Debatten von NGOs wurde auch deutlich, dass zivilgesellschaftliche Organisationen künftig über den Umwelt-, Arbeits- und Menschenrechtsbereich hinaus weitere Implikationen transnationaler Wirtschaftsaktivitäten in den Blick nehmen müssen. TNCs haben zum Beispiel erheblichen Einfluss auf die Steuerpolitik und die Einnahmesituation von Staaten und können die staatliche Handlungsfähigkeit, wie zum Beispiel im Umwelt- und Sozialbereich, erheblich einschränken.

Ausländische Investoren haben auch Einfluss auf die Zahlungsbilanz eines Landes, auf seine Industriepolitik und selbst auf die öffentliche Meinung und die Politik insgesamt. Freiwillige Selbstverpflichtungen der Wirtschaft befassen sich mit diesen „Nebeneffekten“ ihrer Aktivitäten in der Regel nicht. Und auch die Kampagnen von NGOs und Gewerkschaften haben sie in der Vergangenheit zu wenig beachtet. Das Eintreten für internationale Unternehmensregeln ,,jenseits der Freiwilligkeit" erfordert daher auch eine Schwerpunktverlagerung in den Strategien von NGOs und Gewerkschaften.

\section{Anmerkungen}

(1) Dieser Artikel ist ein überarbeiteter Auszug aus: Uwe Kerkow/ Jens Martens/ Tobias Schmitt: Die Grenzen der Freiwilligkeit. Handlungsmöglichkeiten und Erfahrungen von NGOs und Gewerkschaften bei der Anwendung freiwilliger Selbstverpflichtungen der Wirtschaft, WEED-Arbeitspapier, Bonn/Berlin 2003.

(2) United Nations Commission on Sustainable Development: Responsible Entrepreneurship. NGO Perspectives and Recommendations, Background Paper No. 3, New York 1998. Download unter: www.un.org (3) United Nations Subcommission on the Promotion and Protection of Human Rights: Draft Norms on Responsibilities of Transnational Corporations and Other Business Enterprises with regard to Human Rights, Genf, 2002.

(4) Europäisches Parlament: Entschließung zu EUNormen für in Entwicklungsländern tätige europäische Unternehmen im Hinblick auf die Entwicklung eines europäischen Verhaltenskodex, Strassburg 1999.

\section{Der Autor}

Jens Martens ist Leiter des Programmbereichs Internationale Umwelt- und Entwicklungspolitik bei Weltwirtschaft, Ökologie \& Entwicklung (WEED) in Bonn. Kontakt: WEED, Bertha-von-Suttner-Platz 13, 53111 Bonn, Tel. 0228-7661312,

E-Mail: jens.martens@weed-online.org 
(c) 20I0 Authors; licensee IÖW and oekom verlag. This is an article distributed under the terms of the Creative Commons Attribution Non-Commercial No Derivates License (http://creativecommons.org/licenses/by-nc-nd/3.o/), which permits unrestricted use, distribution, and reproduction in any medium, provided the original work is properly cited. 